outcomes and factors contributing to the deaths. An epidemiological assessment tool was given to paediatricians to identify their practices in care of children with tetanus. A survey among 1000 caregivers in the Idi-Araba community assessed knowledge of- and preventives practices of tetanus in the community.

Data was analysed using the SPSS version 22, demographic, clinical and social data were presented using descriptive statistics in the form of frequency tables, continuous data was presented as means \pm standard deviation. Associations were determined using independent t-tests. Statistical significance was (p-values) set at $\leq 0.05$.

Results There were 102 tetanus admissions between September 2012 and 2017. Mean age at admission was $9.14 \pm 3.65$ years. Although $63.26 \%$ of the children had been previously vaccinated according to the National program of immunization, mortality rates for tetanus was $49.26 \%$.

Several $(65.26 \%)$ health care professionals lacked adequate information about prevention of- or management of a child with tetanus.

Among caregivers, 48.21\% had never heard of tetanus, $50.21 \%$ of those who were aware had never vaccinated their wards and poor preventive practices was seen in $70.46 \%$ of caregivers. Only $12 \%$ of community children received booster doses after 5 years of age.

Conclusion Despite the nationwide policy to immunise all children with 3 doses of tetanus before the age 1, in Idi-Araba community there is a problem of implementation.

In children who were vaccinated according to the national schedule, Majority still did not get booster doses at school entry points which leaves them at risk.

If we hope to reduce tetanus mortality, there is a need to educate health care professionals and caregivers and ensure proper establishment and implementation of appropriate immunisation practices.

\section{G606(P) CLINICAL IMPACT OF RAPID IDENTIFICATION OF PATHOGENS IN CSF OF GENERAL PAEDIATRIC PATIENTS}

${ }^{1}$ A Riordan, ${ }^{2} \mathrm{~L}$ Wade, ${ }^{2} \mathrm{~L}$ Waugh, ${ }^{3} \mathrm{~F}$ Shaw. ${ }^{1}$ Paediatric Infectious Disease, Alder Hey Children's NHS FT, Liverpool, UK; ${ }^{2}$ General Paediatrics, Alder Hey Children's NHS FT, Liverpool, UK; ${ }^{3}$ Microbiology, Alder Hey Children's NHS FT, Liverpool, UK

\subsection{6/archdischild-2020-rcpch.520}

Rapid diagnosis and treatment of meningitis and encephalitis are advised to help reduce morbidity and mortality. However, few children treated for suspected central nervous system (CNS) infection have confirmed infection. FilmArray ${ }^{\circledR}$ Meningitis/Encephalitis (FA- M/EP) Panel is a multiplex PCR system that can rapidly test for common CNS pathogens.

We aimed to assess the clinical impact of FA-M/EP in general paediatric patients.

From 6th June 2016 FA-M/EP was performed on cerebrospinal fluid (CSF) when requested by the general paediatric consultant. Following an interim review the following changes were made:

- FA-M/EP was not performed on CSF that was visibly blood stained

- FA-M/EP was only performed if enough sample was available to send for confirmatory vial PCR.
- $\mathrm{FA}-\mathrm{M} / \mathrm{EP}$ was performed in in a separate room in the laboratory.

Data was collected for a further 12 months following these changes (October 2016 - October 2017).

Results FA-M/EP was performed on 43 children during JuneOct 2016.

Results of FA-M/EP were helpful in managing $16(37 \%)$ children - see table 1 . In four children results (9\%) FA-M/EP led to prolonging of hospital admission (HSV, pneumococcus, Haemophilus), increasing the length of stay by 17 days. No bed days were saved overall in this period.

FA-M/EP was performed on 63 samples from 59 children during the next 12 months (Oct 2016 - Oct 2017).

Results of FA-M/EP were helpful in managing 30 (48\%) episodes; saving 25 Bed days for 13 episodes. In one child results from FA-M/EP led to prolonging hospital admission by 2 days (Varicella).

All positive results by FA-M/EP for HSV1 HSV2, Varicella zoster virus, Haemophilus and pneumococcus did not correlate with the clinical features and were likely false positives.

\begin{tabular}{|c|c|c|c|c|c|}
\hline FA-M/EP result & $\begin{array}{l}\text { Prolonged } \\
\text { admission }\end{array}$ & $\begin{array}{l}\text { De-escate } \\
\text { abiots }\end{array}$ & $\begin{array}{l}\text { Stop } \\
\text { aiots }\end{array}$ & $\begin{array}{l}\text { Early } \\
\text { discharge }\end{array}$ & $\begin{array}{l}\text { ?False } \\
\text { positive }\end{array}$ \\
\hline Enterovirus & & & 15 & 4 & 1 \\
\hline Parechovirus & & 1 & & & \\
\hline HHV6 & & 1 & & & \\
\hline HSV1 & 1 & & & & 3 \\
\hline HSV2 & 1 & & & & 1 \\
\hline $\mathrm{H}$ influenzae & 1 & & & & 2 \\
\hline Pneumococcus & 1 & & & & 2 \\
\hline Varicella & 1 & & & & 2 \\
\hline Negative & & 17 & 6 & & \\
\hline
\end{tabular}

Conclusions FA-M/EP was useful in rapid detection of enteroviral meningitis and ruling out HSV encephalitis. However false positive results for HSV, VZV, Haemophilus and pneumococcus led to prolonged admissions. In general paediatric patients with a low risk of meningitis/encephalitis rapid tests which give false positive results may lead to unnecessary treatment and prolonged admissions. These tests should be used with caution in this population.

\section{G607(P) ANTIBIOTIC CHOICE IN COMMON PAEDIATRIC EAR, NOSE AND THROAT INFECTIONS: A GUIDE FOR PAEDIATRICIANS}

I Salem. Paediatrics, St George's University Hospitals NHS FT, London, UK

\subsection{6/archdischild-2020-rcpch.521}

Aims Paediatric Ear, Nose and Throat (ENT) conditions are some of the most common presentations to the health service. Despite their prevalence, the treatment choice in children can often be unclear to those outside the ENT field with antibiotic choice often dependent on consultant preference and experience. There are currently few UK-based ENT treatment guidelines available, although local hospitals have formed their 
own, and there is much debate over the most suitable treatment type for different conditions. The main objective of the work was to form a regional guideline for common inpatient ENT conditions to help simplify current hospital and general practice protocols.

Methods We decided to review the past 5 years of ENT admissions who required hospital admission and antibiotics within our trust. This was completed through populating a list of patients using coded hospital data specifically looking at the different types of conditions, choice of medication, treatment length and where possible culture sensitivities. The aim was to them compare them with local and international protocols. A systematic literature search was completed alongside the work.

Results In the 5 year period, 3265 patients were seen as an inpatient under ENT and of those 1103 patients received antibiotics. The most common presentations were acute mastoiditis, tonsillitis, neck abscess (superficial and deep) and acute otitis media and externa infections. The most common antibiotic used was Co-amoxiclav of different concentrations with 1245 courses supplied with Co-amoxiclav 1000/200 being the most common. The next most used was Benzyl-penicillin IV with 189 courses given.

Conclusion Certain types of antibiotics are used more commonly within paediatric ENT cases that may not be in line with antimicrobial stewardship. Despite some protocols suggesting other antibiotics as first line, medications that are easier for the patient to take and administered less often are used more commonly despite being more broad-spectrum. Clearer guidelines for ENT doctors, general paediatricians and general practitioners are required to ensure the most appropriate antibiotics are prescribed. Further research is needed to identify which antibiotics are most suitable for specific ENT cases before a national guideline can be drawn up.

\section{G608(P) SCHISTOSOMIASIS SCREENING IN UNACCOMPANIED CHILD REFUGEES}

${ }^{1} \mathrm{~A}$ Mears, ${ }^{2} \mathrm{~S}$ Eisen, ${ }^{2} \mathrm{~J}$ Cohen. ${ }^{1}$ Paediatric Urology, University College London Hospitals NHS FT, London, UK; ${ }^{2}$ Paediatrics, University College London Hospitals NHS FT, London, UK

\subsection{6/archdischild-2020-rcpch.522}

Aims To assess our strategy for diagnosing schistosomiasis in unaccompanied child refugees in the UK. Schistosomiasis is an easily treatable infection with significant risk of long term complications if not treated.

Methods A retrospective analysis of a prospectively collected database of a health screening clinic for unaccompanied child refugees.

Urinalysis, stool microscopy and full blood count with serum save are performed for all refugee young people attending our clinic. Schistosomiasis serology is requested if microscopic haematuria or eosinophilia are found and stool and urine are negative for ova. Schistosomiasis is diagnosed on positive serology or stool/urine microscopy positive for ova. Treatment is single dose praziquantel.

Our incidence of diagnosed schistosomiasis is compared to published country prevalence data.

Results 232 unaccompanied refugees age 16-18 years (median 17 years) were assessed.

$44 / 232$ had eosinophilia $>0.4 \times 10^{9} / \mathrm{L}$ (range $0.4-2.02 \times$ $\left.10^{9} / \mathrm{L}\right)$. Of these 9 had stool positive for S.Mansoni ova, 7 had serum-positive schistosomiasis, 12 had other parasites diagnosed on stool microscopy and 16 tested negative.

$20 / 232$ had microscopic haematuria on urinalysis. Only $1 /$ 20 had S.Haematobium ova on microscopy. One had negative microscopy but had eosinophilia and serum-positive schistosomiasis.

$17 / 217(7.8 \%)$ patients who provided stool samples tested positive for S.Mansoni ova, 8/17 did not have eosinophilia.

Therefore a total of $25(10.7 \%)$ young refugees (24 male, 1 female) tested positive for schistosomiasis: 1 on urine microscopy, 17 on stool and 7 on serology. Countries of origin were Eritrea (10), Sudan (9) and Ethiopia (6). 10/63 (15\%) of Eritrean refugees tested positive, 9/41 (22\%) of Sudanese refugees and 6/26 (23\%) of Ethiopian refugees. Published country population prevalences of schistosomiasis are $41 \%$ for Eritrea and 34\% for Sudan.

One further Eritrean patient who had normal stool, urine and eosinophils and was discharged, subsequently attended a Tropical Medicine clinic and tested serum-positive for schistosomiasis.

Conclusion We are diagnosing significant rates of schistosomiasis. However, the higher published prevalences for the countries of origin suggest cases may be being missed with our current screening method which relies heavily on eosinophilia and microscopy.

Our screening strategy will therefore be adjusted to include schistosomiasis serology on all young refugees from subSaharan Africa. We will prospectively assess the impact of this on our diagnosis rate.

\section{G609(P) PERTUSSIS SEROIMMUNITY IN MOTHER-NEONATE PAIRS IN EGYPT}

${ }^{1}$ E Hossny, ${ }^{1} \mathrm{D}$ El-Ghoneimy, ${ }^{2} \mathrm{DA}$ Soliman, ${ }^{3} \mathrm{~A}$ Taher, ${ }^{4} \mathrm{AG}$ Sherif. ${ }^{1}$ Paediatric Allergy and Immunology, Ain Shams University, Cairo, Egypt; ${ }^{2}$ Clinical Pathology, Ain Shams University, Cairo, Egypt; ${ }^{3}$ Ministry of Health, Cairo, Egypt; ${ }^{4}$ Paediatrics, Mataria Teaching Hospital, Cairo, Egypt

\subsection{6/archdischild-2020-rcpch.523}

Background Despite the widespread availability of 2 classes of effective vaccines, whole cell and acellular, pertussis has resurged as a serious public health problem. We sought to investigate the pertussis immune status of mother-neonate pairs in our country where pertussis vaccination is obligatory.

Methods This cross-sectional study was carried during the period from June 2012 to April 2014 and it included 75 healthy full term neonates and their mothers. The enrolled mothers were ensured to be free of any chronic illness and not receiving immunosuppressive drugs. Serum pertussis IgG was measured in all enrolled subjects. A positive titre was defined as $>24 \mathrm{U} / \mathrm{ml}$.

Results All mothers (100\%) had their vaccination according to the Expanded Program of Immunization (EPI) in the health care offices of the Egyptian Ministry of Health. Their newborns were all full terms. Positive pertussis IgG levels were detected in 69 of the mothers (92\%) and in 63 of their newborns (84\%). Serum pertussis IgG titers among the neonates showed a significant positive correlation with the maternal titers $(\mathrm{P}=0.00001)$. Higher rates of pertussis seroimmunity were observed among mothers who are residents in urban and suburban areas as compared to those living in rural areas $(\mathrm{P}<0.05)$. 